\title{
Syncope as a presenting feature of hindbrain herniation with syringomyelia
}

\author{
F HAMPTON, BERNARD WILLIAMS, LA LOIZOU \\ From The Midland Centre for Neurosurgery and Neurology, Smethwick, Warley, UK
}

SUMMARY Syncope is rare as a presenting symptom of syringomyelia. Three cases are described in which syncope brought the patient to hospital and in each case syringomyelia was subsequently diagnosed and treated. The suggestion is made that impaction of hindbrain hernia or the Chiari malformation may be a causative mechanism of loss of consciousness in such cases; the three examples presented all did well after posterior fossa surgery to decompress the hindbrain hernia.

Chronic hindbrain herniation often occurs in association with syringomyelia and either may be responsible for the predominant symptomatology. Here three cases presenting with syncope are described.

\section{Case reports}

Case 1 A 52-year-old woman was admitted to hospital after a blackout followed by prostration with headache and vomiting. She had had several episodes of loss of consciousness in the preceding year, occurring without warning whilst standing, and followed by headache. She noted that coughing and straining produced an unpleasant "pounding" in the head and she had recently developed vertigo on bending, but these did not precede the attacks. She reported numbness in her right hand for at least twenty years. On examination, she was pale and still with neck stiffness and photophobia. There was reduced sensation to pinprick and light touch in the right hand and shoulder and a small patch outside the right foot. Temperature sensation was reduced in both arms. The small hand muscles on the right were wasted. Lumbar puncture revealed normal cerebrospinal fluid at a pressure of $50 \mathrm{~mm}$ CSF. Her symptoms then worsened so that the patient was bed bound and was not able to stand or even sit without discomfort until after operation. Skull radiographs showed mild basilar invagination. Air myelography revealed tonsillar descent and a thin collapsed cervical cord characteristic of syringomyelia. Pressure measurements in ventricular and lumbar CSF demonstrated dissociation after coughing (fig a). The major part of the dissociation was shown in the post-

Address for reprint requests: Bernard Williams, The Midland Centre for Neurosurgery and Neurology, Holly Lane, Smethwick, Warley, West Midlands B67 7JX, UK.

Received 26 March 1982 and in revised form 4 June 1982. Accepted 28 June 1982
Valsalva rebound and the presence of this indicates that the autonomic system was functioning in control of blood pressure. A cranio-vertebral decompression was performed on 16 October and following this her symptoms improved. Subsequent pressure studies were normal (fig b). She recovered well from the operation and all symptoms were improved except the sensory changes in the arm. About two weeks after getting home her headache suddenly recurred on the top of the head and she began to suffer from intermittent worsening of the clumsiness and loss of sensation in the right hand. She had occasional attacks of giddiness. After the sudden return of headache she progressively improved in that respect until April 1982 when she had another attack of unconsciousness lasting for half an hour. When she awoke she had severe headache, neck stiffness and photophobia. The syringomyelic features were unchanged. Once more she went through a period of being unwell when she tried to stand. Reimpaction of the hindbrain hernia was thought to be the cause; lumbar puncture and reassessment of pressures was not done because of fear of exacerbating her problems. She was nursed head down on complete bed rest for a week and this once more produced resolution of her headache and giddiness. Her blood pressure was low throughout the illness, usually being between 100 and $110 \mathrm{~mm} \mathrm{Hg}$ systolic but no orthostatic hypotension was ever demonstrated, blood pressure when standing usually being higher than when she was lying.

Case 2 A 20-year-old man presented with a three-month history of headaches and blackouts. The headaches were occipital, worse on standing, and lasted a few minutes each in bouts over several hours. His blackouts were always preceded by headache. He had burns on his right hand which he attributed to his work with hot metal and claimed to have felt. His only other sign was patchy sensory impairment to pinprick and light touch on the right side. Skull radiographs showed widening of the space between 
(a)

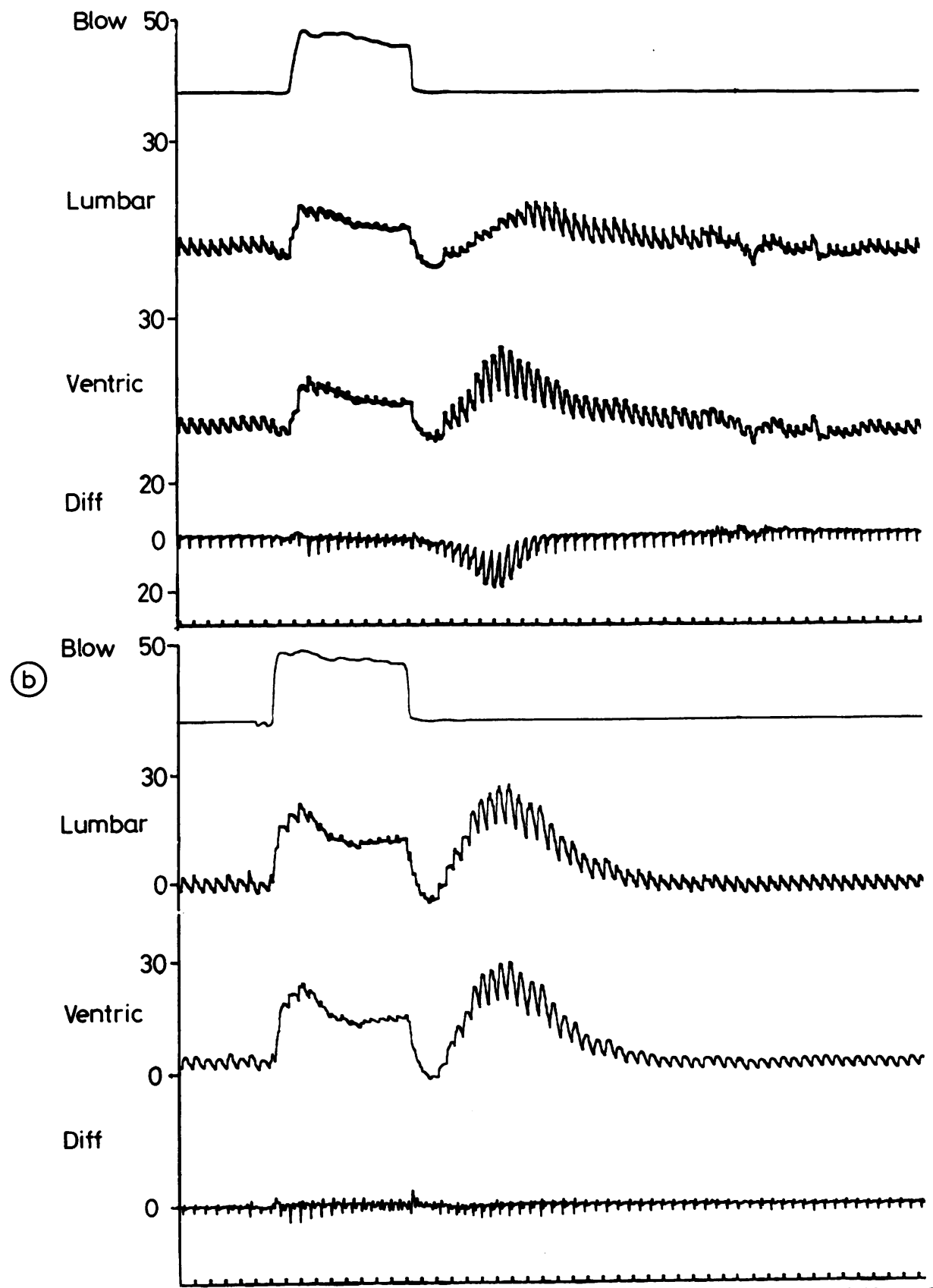

Fig (a) Pre-operative pressure recordings from Case 1 during and after forcible expiration to around $40 \mathrm{~mm} \mathrm{Hg}$ for about $8 \mathrm{~s}$. The differential trace shows a pressure of nearly $20 \mathrm{~mm} \mathrm{Hg}$ between the head and the spine, forcing the hindbrain hernia and medulla into the foramen magnum; (b) post-operative recordings showing that the hindbrain hernia is no longer being impacted in the foramen magnum. 
the foramen magnum and the arch of $\mathrm{C} 1$, with no basilar invagination. A myelogram demonstrated marked tonsillar descent and was followed by prostration with headache and increased right-sided numbness. Right arm reflexes were lost. On blowing into a sphygmomanometer to $\mathbf{4 0}$ $\mathrm{mm} \mathrm{Hg}$ the headache was worsened. After eight days of strict bed rest he improved remarkably and no signs remained. Blood pressure was normal on lying and standing. Pressure studies showed little dissociation, but headache could not be induced. Craniovertebral decompression was performed in December 1980 and he remained symptom free thereafter. He was free from complaints in the spring of 1982 .

Case 3 A 21-year-old man was straining to pass urine when he was affected by a headache at the vertex. He became unconscious until he was being transferred into an ambulance. A second similar episode one week later was accompanied by paraesthesiae over the right arm and face. $\mathrm{He}$ was extensively investigated at that time; carotid angiography showed filling of the posterior circulation retrogradely down the basilar artery. The cause of the syncope was not found at that stage; orthostatic hypotension and hypoglycaemia were not confirmed. Subsequently for about six months he had relapsing and remitting paraesthesiae of the right side, particularly the hand, precipitated by coughing. He was then asymptomatic for three years but at the age of 25 he began to have occipital and biparietal headache precipitated by getting up in the morning and by straining. He developed bilateral features of syringomyelia affecting the arms and at the age of 27 he had vertical nystagmus, weakness and deformities of both hands and absent arm jerks and mildly spastic legs with severe dissociated sensory loss over the arms and painless burns. The syncope did not recur. The blood pressure was normal on lying and standing. Pressure studies of the intracranial compartments showed dissociation typical of hindbrain hernia similar to those in fig a. The return to equality was helped by tilting the head backwards. Craniovertebral decompression was carried out in May 1980. Three months after surgery he was remarkably better with improved mobility and power of the left arm in particular with resolution of the sensory loss and return of the tendon reflexes to the arms. The nystagmus was still present and he remained prone to mild headaches after heavy coughing. No further syncope had occurred. Some degree of pressure dissociation was still detectable but was improved by Torkildsen's operation of ventriculo-cisternostomy which the patient claimed improved his symptoms further; but this was a subjective evaluation and no objective gains could be detected on examination. He remained substantially unchanged in May 1982 preserving his improvement in sensation and arm mobility but still complaining of neck pain chiefly on the left side. There were no further faints.

\section{Discussion}

Headaches and syncope have been described as the major feature in chronic hindbrain herniation, usually following coughing, sneezing or some other exertion. ${ }^{1-3}$ One explanation for cough headache is craniospinal pressure dissociation. Increasing intrathoracic and abdominal pressure causes CSF from the spinal subarachnoid space to move intracranially and relaxation is accompanied by flow back into the spinal compartment. When a hindbrain hernia is present the temporarily higher intracranial pressure can impact the hernia at the foramen magnum resulting in a long lasting pressure difference. ${ }^{45}$ The "half life" of the pressure difference between the head and the spine is, in the normal person, of the order of 0.1 second and may extend to $0.4 \mathrm{sec}$ ond in cases with no gross abnormality. In one case a difference of $100 \mathrm{~mm} \mathrm{Hg}$ falling over 40 seconds has been shown.' The mechanism of jamming the hindbrain hernia into the foramen magnum could be responsible for syncope by interfering with the medullary baroreceptor reflex or by dysfunction of the midbrain reticular activating system. ${ }^{2}$ Pressure differences of the order shown by this pressure testing are presumably capable of making parts of the hindbrain temporarily ischaemic.

An alternative explanation of syncope in syringomyelia is failure of the autonomic system because of excavation of the lateral horns of the grey matter of the thoracic cord producing what is in effect a sympathectomy and thus causing postural hypotension. Such an explanation seems improbable in these cases since the post-Valsalva rebound was present in all the cases and no postural hypotension was demonstrated. Also the unconsciousness did not seem to be closely related to getting up or to be reproducible by this manoeuvre.

In our cases it is not clear what might have precipitated each episode. However, some features support craniospinal pressure dissociation. Case 1 had become severely constipated due to avoiding straining, although denying headache or syncope related to it. She had a small, but definite pressure dissociation after coughing, and on presentation she had a low lumbar CSF pressure. Lumbar puncture resulted in further impaction and was followed by worsening of symptoms. The subsequent relapse is of course, disappointing and may reflect the observation that craniovertebral decompression does not always correct pressure dissociation. ${ }^{5}$ Slumping of the lower cerebellum into the decompression is not uncommon even after careful surgery and management thereafter may be difficult. A Torkildsen procedure as used in Case 3 may be useful but it's effectiveness for correction of failure of operation to rectify cranio-spinal pressure dissociation remains unproven.

Although no dissociation was shown in Case 2 the symptoms could not be reproduced at the time that the testing was done and earlier on in the illness an increased intrathoracic pressure was certainly followed by severe headaches, as was the myelogram. 
That all cases were improved at least temporarily by an operation designed to discourage further impaction is the strongest indication for considering hindbrain herniation as one of the causes of unexplained syncope. Syncope is not usually considered as a feature of syringomyelia, although Barnett, Foster and Hudgson reported two cases with "drop attacks" in their monograph, consciousness was retained. ${ }^{6}$ It is suggested that in undiagnosed syncope a search for features of syringomyelia may sometimes be rewarding.

\section{References}

' Williams B. Cough headache due to craniospinal pressure dissociation. Arch Neurol 1980;37:226-30.

${ }^{2}$ Dobkin BH. Syncope in the adult Chiari anomaly. Neurology (Minneap) 1978;28:718-20.

${ }^{3}$ Corbett JJ, Butler AB, Kaufman B: "Sneeze syncope", basilar invagination and Arnold-Chiari type 1 malformation. $J$ Neurol Neurosurg Psychiatry 1976;39:381-4.

${ }^{4}$ Williams B. Simultaneous cerebral and spinal fluid pressure recordings. 1. Technique, physiology and normal results. Acta Neurochir 1981;58:167-85.

${ }_{5}^{5}$ Williams B. Simultaneous cerebral and spinal fluid pressure recordings. 2. Cerebrospinal dissociation with lesions at the foramen magnum. Acta Neurochir 1981;59:123-42.

- Barnet HJM, Foster JB, Hudgson P. Syringomyelia. London: WB Saunders, 1973. 